\title{
Migration of protoplanets in radiative discs
}

\author{
W. Kley and A. Crida \\ Institut für Astronomie \& Astrophysik, Universität Tübingen, Auf der Morgenstelle 10, 72076 Tübingen, Germany \\ e-mail: wilhelm.kley@uni-tuebingen.de
}

Received 23 April 2008 / Accepted 18 June 2008

ABSTRACT

\begin{abstract}
Context. In isothermal discs, the migration of protoplanets is directed inwards. For small planetary masses, the standard type I migration rates are so high that this can result in an unrealistic loss of planets into the stars.

Aims. We investigate the planet-disc interaction in non-isothermal discs and analyse the magnitude and direction of migration for an extended range of planet masses.

Methods. We performed detailed two-dimensional numerical simulations of embedded planets including heating/cooling effects as well as radiative diffusion for realistic opacities.

Results. In radiative discs, small planets with $M_{\text {planet }}<50 M_{\text {Earth }}$ migrate outwards at a rate comparable to the absolute magnitude of standard type I migration. For larger masses, the migration is inwards and approaches the isothermal, type II migration rate.

Conclusions. Our findings are particularly important for the first growth phase of planets and ease the problem of too rapid inward type I migration.
\end{abstract}

Key words. stars: planetary systems: formation - accretion, accretion disks

\section{Introduction}

Planets form in discs surrounding young stars. The growing protoplanets undergo an embedded phase where the gravitational interaction with the ambient gaseous disc produces a change in its orbital elements. For protoplanets of masses below about 30 Earth masses, the disc is not disturbed too strongly and the interaction can be analysed using a linear approximation. By calculating the total disc torques acting on the planet, it is found at these small masses that the semi-major axis is reduced, i.e. an inward migration occurs (Goldreich \& Tremaine 1979; Ward 1997; Tanaka et al. 2002; Tanaka \& Ward 2004). The inward drift of this type I migration was measured to be extremely rapid, and the planets were found to be lost before they could grow to become larger objects (Korycansky \& Pollack 1993). This problem was highlighted by comparing population synthesis models with the characteristics of observed planetary systems (Alibert et al. 2004; Ida \& Lin 2008). To avoid this rapid phase of inward migration, alternative scenarios were sought. In a turbulent disc, migration occurs stochastically with inwards and outwards phases that deaccelerate the migration (Nelson 2005). Departures from the linear regime at around 10-20 $M_{\text {Earth }}$ can also reduce the inward migration (Masset et al. 2006a). However, both processes are insufficient to solve the problem. The planet trap scenario for halting planetary migration (Masset et al. 2006b) requires a positive density gradient, which in general may not be present.

To simplify calculations, almost all analytical and numerical studies of the planet-disc interaction process have focussed on isothermal discs, for which the temperature is a function of position inside the disc. Early work on non-isothermal discs focussed on high mass embedded planets and did not find a strong effect on migration (D'Angelo et al. 2003; Klahr \& Kley 2006). Using fully three-dimensional radiative calculations of an embedded small mass planet, Paardekooper \& Mellema (2006) demonstrated in a very important work that migration can be deaccelerated significantly or even reversed, when thermal effects are included. Subsequent analysis indicated that this behaviour was related to a radial entropy gradient in the flow (Baruteau \& Masset 2008; Paardekooper \& Mellema 2008). Paardekooper \& Papaloizou (2008) showed that for small planet masses, the combination of radiative and viscous diffusion could permit longterm unsaturated positive torques and outward migration.

In this letter, we investigate this possibility in more detail, for a range of planetary masses, which enable us to estimate its effect on the long term evolution of the planet. For that purpose, we perform two-dimensional numerical hydrodynamical simulations of embedded planets in radiative discs. A method to treat the three-dimensional radiative transfer approximately in these 2D simulations is outlined in the following section. Our measurements of the migration rate for various masses (in Sect. 3) indicate that, for masses smaller than about $50 M_{\text {Earth }}$, the torques remain unsaturated in the long term and migration is indeed directed outwards, while larger planets drift inwards. The consequence for the migration process and the overall evolution of planets in discs is discussed.

\section{Physical modelling}

The protoplanetary disc is treated as a two-dimensional, nonself-gravitating gas that can be described by the Navier-Stokes equations. The embedded planet is modelled as a point mass that orbits the central star on a fixed, circular orbit. For the planetary potential, we use a smoothing length of $\epsilon=0.6 H$, where $H$ is the vertical scale height of the disc. To calculate the gravitational torques acting on the planet, we apply a tapering function to exclude the inner parts of the Hill sphere of the planet, where the transition lies at 0.8 Hill radii (see Crida et al. 2008, Fig. 2). 


\subsection{Energy equation}

In the present setup, we utilise fully radiative models with an improved thermodynamic treatment that uses the thermal energy equation in the following form:

$\frac{\partial \Sigma c_{\mathrm{v}} T}{\partial t}+\nabla \cdot\left(\Sigma c_{\mathrm{v}} T \boldsymbol{u}\right)=-p \nabla \cdot \boldsymbol{u}+D-Q-2 H \nabla \cdot \boldsymbol{F}$,

where $\boldsymbol{u}=\left(u_{r}, u_{\varphi}\right)$ is the two-dimensional velocity, $\Sigma$ the density, $p$ the pressure, $T$ the (mid-plane) temperature of the disc, and $c_{\mathrm{V}}$ is the specific heat at constant volume. On the right hand side, the first term describes compressional heating, $D$ the (vertically averaged) dissipation function, $Q$ the local radiative cooling from the two surfaces of the disc, and $\boldsymbol{F}$ denotes the two-dimensional radiative flux in the $(r, \varphi)$-plane. For all models, $H$ is calculated from the soundspeed as $H(r)=c_{\mathrm{S}} / \Omega_{\mathrm{K}}(r)$, where $\Omega_{\mathrm{K}}$ is the Keplerian angular velocity, and $c_{\mathrm{s}}=\sqrt{\gamma p / \Sigma}, \gamma=1.43$ being the adiabatic index. The 2D-pressure is given by $p=R_{\text {gas }} \Sigma T / \mu$ with the mean molecular weight $\mu=2.35$.

To calculate the radiative losses $Q$ (from both sides of the disc), we follow D’Angelo et al. (2003) and Kley et al. (2005),

$Q=2 \sigma_{\mathrm{R}} T_{\mathrm{eff}}^{4}$,

where $\sigma_{\mathrm{R}}$ is the Stefan-Boltzmann constant and $T_{\text {eff }}$ is an estimate of the effective temperature (Hubeny 1990), given by:

$T_{\text {eff }}^{4} \tau_{\text {eff }}=T^{4}, \quad$ with $\quad \tau_{\text {eff }}=\frac{3}{8} \tau+\frac{\sqrt{3}}{4}+\frac{1}{4 \tau}$.

For our two-dimensional disc, we approximate the mean vertical optical depth by $\tau=(1 / 2) \kappa \Sigma$, where for the Rosseland mean opacity $\kappa$ the analytical formulae by Lin \& Papaloizou (1985) are adopted. The radiative transport in the plane of the disc is treated by the flux-limited diffusion approximation where the flux is given by:

$\boldsymbol{F}=-\frac{\lambda c 4 a T^{3}}{\rho \kappa} \nabla T$

where $c$ is the speed of light, $a$ the radiation constant, $\rho=$ $\Sigma /(2 H)$ the mid-plane density, and $\lambda$ the flux-limiter (see Kley 1989).

In the following, we present results of numerical simulations using various sub-parts of the energy equation, Eq. (1). If only the first term on the right hand side is used, the model is called adiabatic; when only the last term is omitted, it is a model with local heating and cooling; the usage of the full energy equation is termed fully radiative. To relate to previous results we also use isothermal models, where a pre-described radial temperature distribution is maintained at a constant and no energy equation at all is solved. In the full version, we note that the radiative treatment (in Eq. (1)) incorporates full 3D effects of the radiative transport, for which the vertical part (z-direction) is taken care of by the local cooling term, $Q$, and the horizontal part through $\boldsymbol{F}$.

\subsection{Reference model}

The two-dimensional $(r-\varphi)$ computational domain consists of a complete ring of the protoplanetary disc centred on the star, extending from $r_{\min }=0.4$ to $r_{\max }=2.5$ in units of $r_{0}=a_{\text {Jup }}=$ 5.2 AU. The mass of the central star is one solar mass, and the total disc mass inside $\left[r_{\min }, r_{\max }\right]$ is $M_{\text {disc }}=0.01 M_{\odot}$. For the present study, we maintain the kinematic viscosity constant at a value of $v=10^{15} \mathrm{~cm}^{2} / \mathrm{s}$, a value that relates to an equivalent
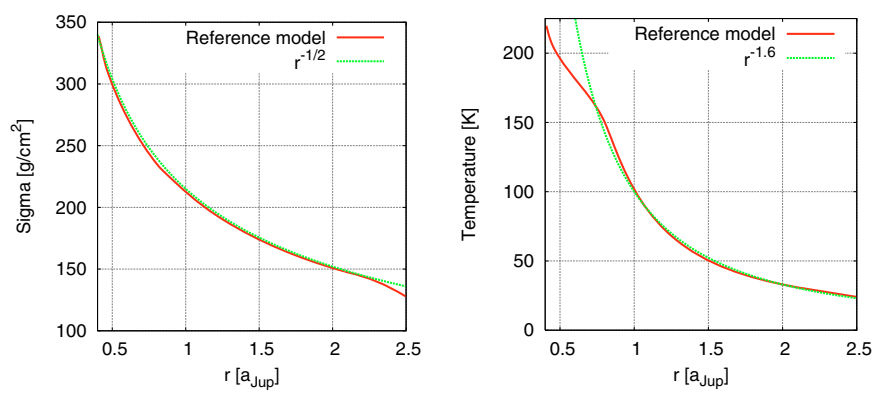

Fig. 1. Density and temperature versus radius for the relaxed reference model. For illustrative purposes, simple comparison power laws have been added.

$\alpha$ at $r_{0}$ of 0.004 for a disc aspect ratio of $H / r=0.05$, where $v=\alpha H^{2} \Omega_{\mathrm{K}}$.

To construct a joint reference model for the different types of approximations to the energy equation (isothermal, adiabatic, etc.), we construct a fully radiative model with no embedded planet, using the above layout.

This model is obtained from an approximate initial state (with $H / r=0.05$ ) that is then relaxed to its equilibrium by performing a time evolution of the disc solving the full NavierStokes equations including energy. At the onset of this process, the initial disc stratification is given by $\Sigma(r) \propto r^{-1 / 2}, T(r) \propto r^{-1}$, and pure Keplerian rotation $\left(u_{r}=0, u_{\varphi}=\left(G M_{*} / r\right)^{1 / 2}\right)$. The evolution towards the equilibrium takes about 150 orbits and the resulting density and temperature distribution is displayed in Fig. 1. The density has a $\Sigma \propto r^{-1 / 2}$ profile that for constant $v$ follows directly from the angular momentum equation. The temperature has a $T \propto r^{-1.6}$ profile, which follows from $Q=D$ for the used opacity. These relations are superimposed on the numerical results in Fig. 1. For small radii (and higher temperatures), the opacity law has a different temperature dependence and the slope $T(r)$ is altered. The relative scale height for this radiative model is not constant but declines with radius. At $r=1$, we find $H / r \approx 0.045$, and at the outer radius $H / r \approx 0.03$.

\subsection{Numerics}

We adopt a rotating reference system, which rotates at the orbital frequency of the planet. For our standard cases, we use an equidistant grid in $r$ and $\varphi$ with a resolution of $128 \times 384$. In an effort to ensure a uniform environment for all models and minimize disturbances (wave reflections) from the radial boundaries, we impose, at $r_{\min }$ and $r_{\max }$, damping boundary conditions where both velocity components are relaxed towards their initial state on a timescale of approximately the local orbital period. As the initial radial velocity vanishes, this damping routine ensures that no mass flows through the boundaries such that the total disc mass remains constant. The angular velocity is relaxed towards the Keplerian values. For the density and temperature, we apply closed radial boundary conditions. In the azimuthal direction, periodic boundary conditions are imposed for all variables.

The numerical details of the used finite volume code (RH2D) relevant for these disc simulations were described by Kley (1999), where we implemented in addition the FARGO method (Masset 2000) that increases the speed of the numerical computation of differentially rotating flows. The energy equation Eq. (1) is solved explicit-implicitly applying operator-splitting. The heating and cooling term $D-Q$ is treated as one sub-step in this procedure (see Günther et al. 2004), and the additional 


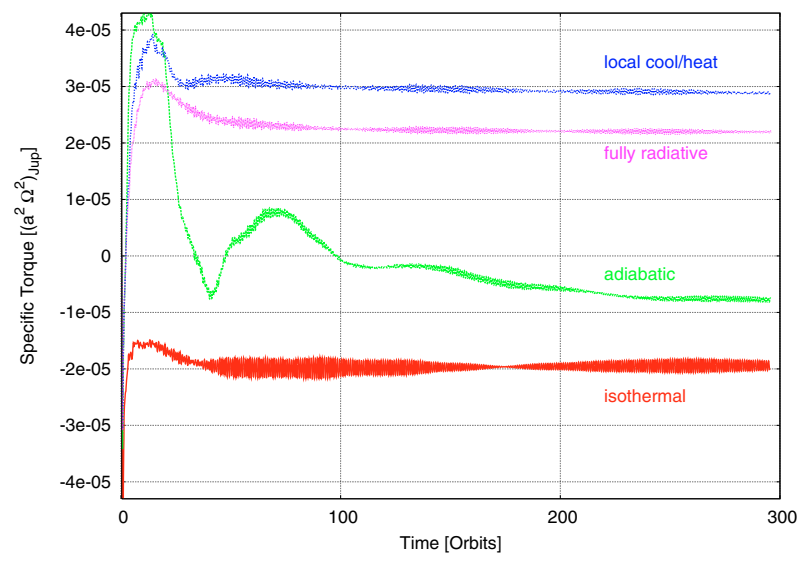

Fig. 2. Time evolution in the specific total torque exerted by the disc on an embedded protoplanet of $20 M_{\text {Earth }}$ for various approximations to the energy equation.

radiative diffusion part in the energy equation is solved by an implicit method to avoid possible time step limitations.

\section{Results}

\subsection{A model with $20 M_{\text {Earth }}$}

To illustrate the influence of the 4 different formulations of the energy equation on the torque, we present a model with an embedded $20 M_{\text {Earth }}$ planet. For this particular mass, a 3D-study, which analysed the effects of an isothermal disc on all orbital elements, measured negative rates of migration as well as of eccentricity and inclination changes (Cresswell et al. 2007). The starting configuration (at $t=0$ ) for the 4 cases was the equilibrated reference model corresponding to the fully radiative case as described above (see Fig. 1). In Fig. 2, we display the time evolution of the torque acting on the planet, for the different formulations of the energy equation. As expected, the isothermal condition leads after about 50 orbital periods to a constant negative torque implying inward migration. The adiabatic model has an initial phase of positive torques, before settling to negative values of about $40 \%$ of the isothermal case. This behaviour was suggested by Baruteau \& Masset (2008) who argued that in the adiabatic case, the torques are unsaturated and positive during the onset phase, while in the long-term, they should converge to saturated negative values.

In contrast, in both radiative models, the torques settle to constant positive values. The fully radiative case yields a $25 \%$ smaller value due to the heat diffusion included in the disc plane. In all cases, we have continued the simulations with double and quadruple resolution. In general, we found (for this planet mass) similar results and a convergence of the torques at about double resolution. Only the model with pure heating/cooling (i.e. no radiative diffusion) requires apparently far higher resolution to achieve convergence, which we attribute to the highly local character of this type of energy equation. The addition of radiative diffusion in the disc plane eases the numerical requirements and at the same time makes the simulations more realistic physically.

\subsection{Variation of the planet mass}

To estimate the influence of the planet mass, we performed a sequence of models with masses ranging from $q=10^{-5}\left(3 M_{\text {Earth }}\right)$ up to $q=10^{-3}\left(M_{\text {Jupiter }}\right)$. The resulting equilibrium specific
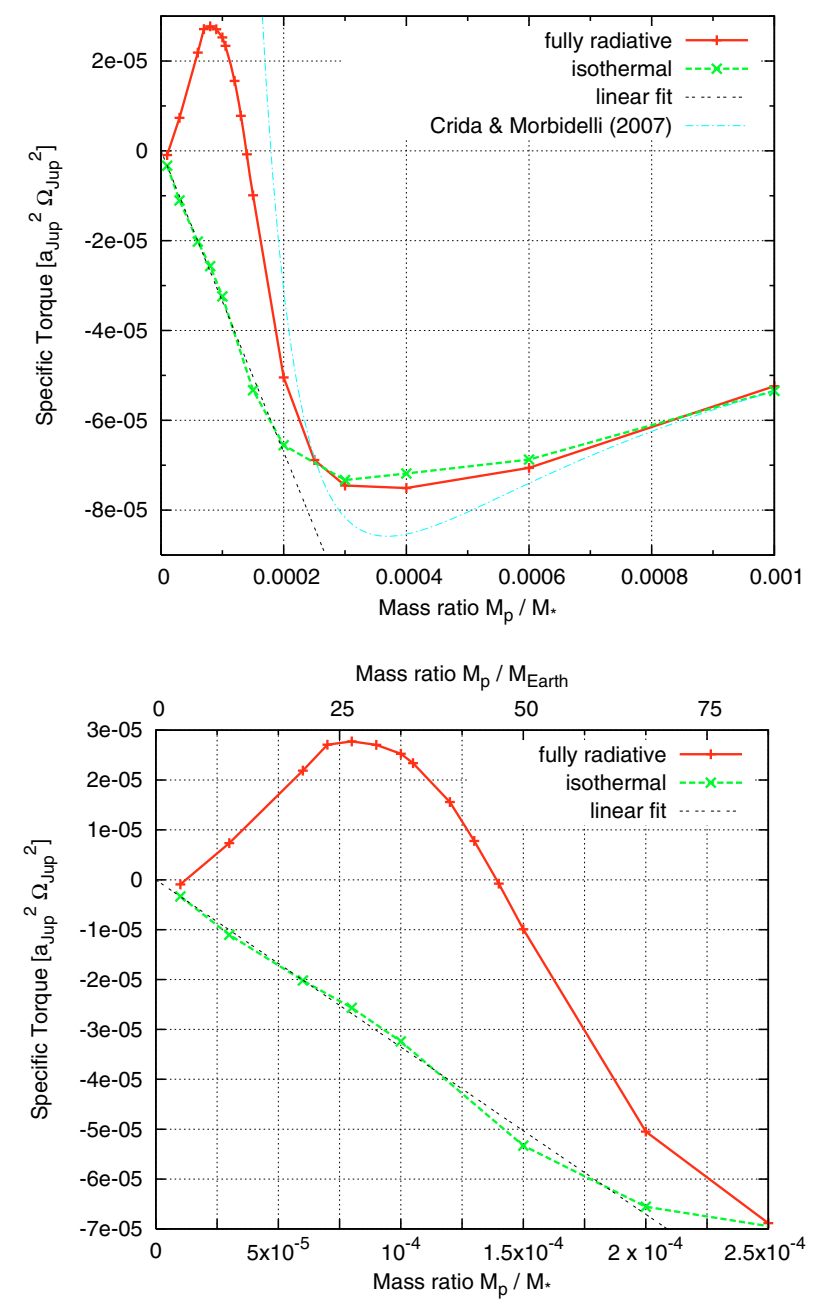

Fig. 3. Comparison of the specific torque exerted on an embedded protoplanet for the isothermal and fully radiative model (thick lines). The lower panel is an enlargement of the upper. Two analytic curves are superimposed (thin lines).

torques are displayed in Fig. 3 for the fully radiative and isothermal case. For small planetary masses up to about $50 M_{\text {Earth }}$, the torque exerted on the planet is clearly positive for the radiative model and becomes negative for masses above this value (see bottom panel). In contrast, for the isothermal models the torques are negative for all masses and follow expected well known results. From the superimposed dotted line, it is clear that the specific torque is proportional to $q$, as predicted by linear theory (e.g. Tanaka et al. 2002).

For large planets $\left(q \geqslant 2.5 \times 10^{-4}\right)$, there is little difference between radiative and isothermal discs, and the torque is well approximated by the model given by Crida \& Morbidelli (2007): the dot-dashed curve in the top panel corresponds to their Eq. (15), where $u_{r}$ is replaced by $\frac{3}{2} \frac{v}{r}$. This model refines the type II migration rate for planets whose gap is not completely empty; therefore, it applies here for $q \gtrsim 3 \times 10^{-4}$ (see the gap profiles in Fig. 5). We find that this model, and type II migration in general, is also valid in radiative discs.

The two-dimensional density distribution for the two cases (isothermal and fully radiative) is displayed in Fig. 4 for a planetary mass of $q=10^{-4}$ (33 $M_{\text {Earth }}$ ) which has the maximum positive torque. In the radiative model, the higher temperature produces slightly larger opening angles of the spiral arms with a reduced density, while the isothermal situation allows for a 

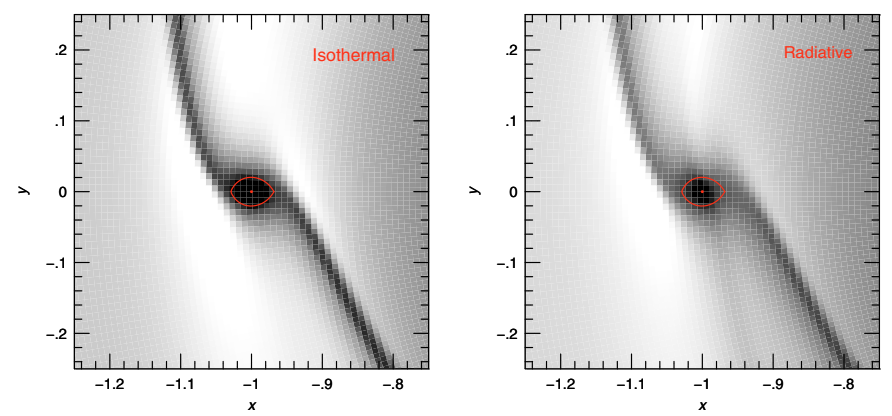

Fig. 4. Two-dimensional grey-scale plot of the density $\left(\propto \Sigma^{1 / 4}\right.$-scaling between 150 and $350 \mathrm{~g} / \mathrm{cm}^{2}$ ) in the vicinity of the planet for the isothermal model (left) and the fully radiative model (right). The planet mass in this case refers to $10^{-4} M_{\odot}$ or $33 M_{\text {Earth }}$. In these plots, we present data for the double resolution models $(256 \times 768)$.
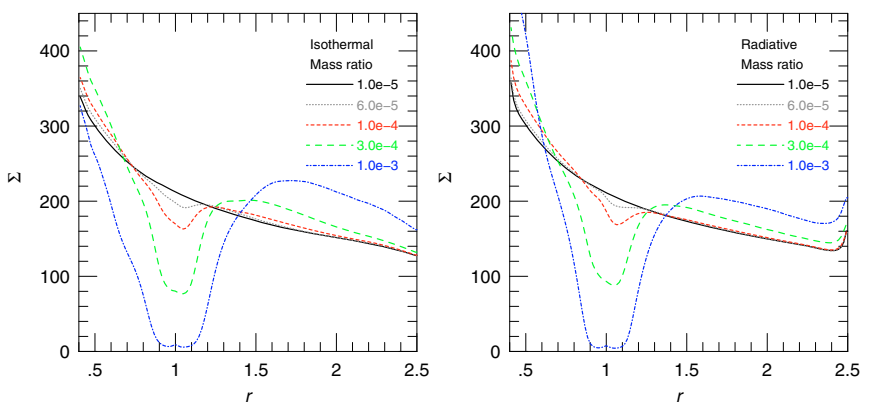

Fig. 5. Azimuthally averaged density for isothermal and radiative cases for different planet masses.

higher mass concentration within the Roche lobe of the planet. As expected from other investigations (Baruteau \& Masset 2008; Paardekooper \& Papaloizou 2008), the inner half of the horseshoe region ahead of the planet (below the planet in Fig. 4) is denser in the radiative case than in the isothermal one, due to the incoming colder gas from the outer half after a U-turn. Symmetrically, the outer half of the horseshoe region is depleted slightly behind the planet. As a consequence of these changes in density, the Lindblad torques are slightly reduced and the corotation torque is more positive. As the net result, we find for this case an outward migration. To maintain the necessary unsaturated corotation torque in the long term, the libration timescales must be comparable to the cooling times (Baruteau \& Masset 2008; Paardekooper \& Papaloizou 2008). For our parameters, we find a cooling time $\tau_{\text {cool }}=T \Sigma c_{\mathrm{v}} / Q$ of about $200 \Omega_{\mathrm{K}}^{-1}$ at the location $r_{\mathrm{p}}$ of the planet. This is comparable to the horseshoe libration time, $\tau_{\text {lib }}=8 \pi r_{\mathrm{p}} /\left(3 \Omega_{\mathrm{K}} x_{\mathrm{s}}\right)$ with the half-width of horseshoe-orbit, $x_{\mathrm{s}}=1.16 r_{\mathrm{p}} \sqrt{q /(H / r)}$ (Baruteau \& Masset 2008). In our case, for $q=10^{-4}$ we find $\tau_{\text {lib }}=150 \Omega_{\mathrm{K}}^{-1}$.

The radial density stratification of the isothermal and radiative cases (Fig. 5) indicate shallower gaps for the latter; in particular, the region inside the planet $(r<1)$ is not cleared as efficiently compared to the isothermal case. Larger planet masses lead to gap openings of similar depths in the isothermal and radiative case. For the largest planet mass, the gap appears to be wider in the isothermal case, possibly due to lower temperatures, but boundary effects (at $r_{\min }$ and $r_{\max }$ ) begin to be visible. For larger planet masses, a radially increased domain is clearly required.

\section{Summary}

We have investigated the migration of planets in discs using twodimensional numerical simulation including heating/cooling effects as well as radiative diffusion.

Using different formulations of the energy equation, we first showed that, for a planet mass of $20 M_{\text {Earth }}$, migration is directed inwards in the isothermal and adiabatic situation, while inclusion of radiative effects leads to an outward migration of the planet. This finding supports the torque reversal mechanism in radiative discs due to corotation effects as suggested by Baruteau \& Masset (2008). A detailed parameter study for planetary masses in the range between $10^{-5}$ and $10^{-3} M_{\odot}$ showed that the effect is limited to planets in the low mass regime, $M_{\mathrm{p}} \leq 50 M_{\text {Earth }}$, where corotation effects are indeed important. Larger mass planets open up gaps in the disc and the migration rate becomes similar to the isothermal case.

Our findings are particularly important for the first growth phase of planets and may ease the problem to too rapid inward type I migration. Depending on the mass accretion rate onto the planet, the growing planetary embryos can spend an extended time span in an outward migration phase and avoid loss into the star. However, close-in planets exist for a range of planetary masses, according to observations. Thus, a significant and long outward migration phase may create new difficulties. Whether this problem really exists can only be answered by following the actual long-term migration of planets through the disc including its mass growth.

Constructing the necessary migration histories of planetary cores, to be used in population synthesis models, requires suitable scaling laws for the migration process as a function of disc parameter $(\Sigma(r), T(r))$ for realistic accretion discs with net mass flow. The present study can be used as a starting point for these larger parameter studies. The inclusion of three-dimensional effects and additional physics (MHD, self-gravity, mass accretion) will make the models even more realistic in the future.

Acknowledgements. Very fruitful discussions with Fredéric Masset and Clément Baruteau are gratefully acknowledged. A. Crida acknowledges the support through the German Research Foundation (DFG) grant KL 650/7.

\section{References}

Alibert, Y., Mordasini, C., \& Benz, W. 2004, A\&A, 417, L25

Baruteau, C., \& Masset, F. 2008, ApJ, 672, 1054

Cresswell, P., Dirksen, G., Kley, W., \& Nelson, R. P. 2007, A\&A, 473, 329

Crida, A., \& Morbidelli, A. 2007, MNRAS, 377, 1324

Crida, A., Sándor, Z., \& Kley, W. 2008, A\&A, 483, 325

D’Angelo, G., Henning, T., \& Kley, W. 2003, ApJ, 599, 548

Goldreich, P., \& Tremaine, S. 1979, ApJ, 233, 857

Günther, R., Schäfer, C., \& Kley, W. 2004, A\&A, 423, 559

Hubeny, I. 1990, ApJ, 351, 632

Ida, S., \& Lin, D. N. C. 2008, ApJ, 673, 487

Klahr, H., \& Kley, W. 2006, A\&A, 445, 747

Kley, W. 1989, A\&A, 208, 98

Kley, W. 1999, MNRAS, 303, 696

Kley, W., Lee, M. H., Murray, N., \& Peale, S. J. 2005, A\&A, 437, 727

Korycansky, D. G., \& Pollack, J. B. 1993, Icarus, 102, 150

Lin, D. N. C., \& Papaloizou, J. C. B. 1985, in Protostars and Planets II, 981

Masset, F. 2000, A\&AS, 141, 165

Masset, F. S., D’Angelo, G., \& Kley, W. 2006a, ApJ, 652, 730

Masset, F. S., Morbidelli, A., Crida, A., \& Ferreira, J. 2006b, ApJ, 642, 478

Nelson, R. P. 2005, A\&A, 443, 1067

Paardekooper, S.-J., \& Mellema, G. 2006, A\&A, 459, L17

Paardekooper, S.-J., \& Mellema, G. 2008, A\&A, 478, 245

Paardekooper, S.-J., \& Papaloizou, J. C. B. 2008 [arXiv: 0804 .4547]

Tanaka, H., \& Ward, W. R. 2004, ApJ, 602, 388

Tanaka, H., Takeuchi, T., \& Ward, W. R. 2002, ApJ, 565, 1257

Ward, W. R. 1997, Icarus, 126, 261 